\title{
The Next Generation Virgo Cluster Survey. XVII. A Search for Planetary Nebulae in Virgo Cluster Globular Clusters
}

\author{
Weijia Sun ${ }^{1,2}$ (10), Eric W. Peng ${ }^{1,2}$ (1) , Youkyung Ko ${ }^{1,2}$ (D) , Patrick Côté $^{3}$ (D), Laura Ferrarese ${ }^{4}$ (D), Myung Gyoon Lee ${ }^{5}$ (iD),

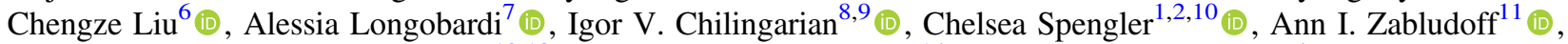 \\ Hong-Xin Zhang ${ }^{12,13}$ (D) , Jean-Charles Cuillandre ${ }^{14}$, and Stephen D. J. Gwyn ${ }^{4}$ \\ ${ }^{1}$ Department of Astronomy, Peking University, Yi He Yuan Lu 5, Hai Dian District, Beijing 100871, People's Republic of China \\ ${ }^{2}$ Kavli Institute for Astronomy \& Astrophysics and Department of Astronomy, Peking University, Yi He Yuan Lu 5, Hai Dian District, Beijing 100871, People's \\ Republic of China \\ ${ }^{3}$ NRC Herzberg Astronomy \& Astrophysics Research Centre, 5071 West Saanich Road, Victoria, BC V9E 2E7, Canada \\ ${ }^{4}$ NRC Herzberg Astronomy and Astrophysics, 5071 West Saanich Road, Victoria, BC V9E 2E7, Canada \\ ${ }^{5}$ Astronomy Program, Department of Physics and Astronomy, Seoul National University, 1 Gwanak-ro, Gwanak-gu, Seoul 08826, Republic of Korea \\ ${ }^{6}$ Department of Astronomy, Shanghai Key Laboratory for Particle Physics and Cosmology, Shanghai Jiao Tong University, People's Republic of China \\ 7 Aix Marseille Universiteé, CNRS, CNES, Laboratoire d'Astrophysique de Marseille UMR 7326, F-13388, Marseille, France \\ ${ }^{8}$ Harvard-Smithsonian Center for Astrophysics, 60 Garden St. Cambridge MA 02138, USA \\ ${ }^{9}$ Sternberg Astronomical Institute, M.V.Lomonosov Moscow State University, Universitetsky prospect 13, Moscow, 119234, Russia \\ ${ }^{10}$ Institute of Astrophysics, Pontificia Universidad Católica de Chile, Av. Vicuña Mackenna 4860, 7820436 Macul, Santiago, Chile \\ ${ }^{11}$ Steward Observatory, University of Arizona, 933 North Cherry Avenue, Tucson, AZ 85721, USA \\ ${ }^{12}$ CAS Key Laboratory for Research in Galaxies and Cosmology, Department of Astronomy, University of Science and Technology of China, Hefei, Anhui 230026, \\ People's Republic of China \\ ${ }^{13}$ School of Astronomy and Space Science, University of Science and Technology of China, Hefei 230026, People's Republic of China \\ ${ }^{14}$ CEA/IRFU/SAp, Laboratoire AIM Paris-Saclay, CNRS/INSU, Université Paris Diderot, Observatoire de Paris, PSL Research University, F-91191 Gif-sur-Yvette \\ Cedex, France \\ Received 2019 April 26; revised 2019 September 29; accepted 2019 September 30; published 2019 November 11
}

\begin{abstract}
The occurrence of planetary nebulae (PNe) in globular clusters (GCs) provides an excellent chance to study lowmass stellar evolution in a special (low-metallicity, high stellar density) environment. We report a systematic spectroscopic survey for the [O III] $5007 \AA$ emission line of PNe in 1469 Virgo GCs and 121 Virgo ultra-compact dwarfs (UCDs), mainly hosted in the giant elliptical galaxies M87, M49, M86, and M84. We detected zero PNe in our UCD sample and discovered one PN $\left(M_{5007}=-4.1 \mathrm{mag}\right)$ associated with an M87 GC. We used the [O III] detection limit for each GC to estimate the luminosity-specific frequency of PNe, $\alpha$, and measured $\alpha$ in the Virgo cluster GCs to be $\alpha \sim 3.9_{-0.7}^{+5.2} \times 10^{-8} \mathrm{PN} / L_{\odot}$. The value of $\alpha$ in the Virgo GCs is among the lowest reported in any environment, due in part to the large sample size, and it is 5-6 times lower than that for the Galactic GCs. We suggest that $\alpha$ decreases toward brighter and more massive clusters, sharing a similar trend as the binary fraction, and the discrepancy between the Virgo and Galactic GCs can be explained by the observational bias in extragalactic surveys toward brighter GCs. This low but nonzero efficiency in forming PNe may highlight the important role played by binary interactions in forming PNe in GCs. We argue that a future survey of less massive Virgo GCs will be able to determine whether PN production in the Virgo GCs is governed by an internal process (mass, density, binary fraction) or if it is largely regulated by the external environment.
\end{abstract}

Key words: galaxies: individual (M87, M49, M86, M84) - globular clusters: general - planetary nebulae: general

\section{Introduction}

Despite of decades of research, the stellar population that forms planetary nebulae $(\mathrm{PNe})$ is still not clear. Traditionally, $\mathrm{PNe}$ are thought to be the circumstellar gas ejected from extreme giant stars at the end of the asymptotic giant branch (AGB) phase, accompanied with an intense mass loss (Abell \& Goldreich 1966). In this view, nearly all stars with a mass between $1.2 M_{\odot}$ and $8 M_{\odot}$ eventually become PNe. However, there remain challenges in explaining the axisymmetric and point-symmetric morphology of $\mathrm{PNe}$, especially young ones (Sahai \& Trauger 1998). Stellar rotation and magnetic fields (García-Segura et al. 1999, 2005) have been proposed to explain this phenomenon, but both suffer from fundamental defects (Nordhaus et al. 2007; García-Segura et al. 2014). Another promising theory involving central star (CS) binarity might be the key to understand PNe formation (e.g., Soker 1997, 2006; Ciardullo et al. 2005; De Marco 2009; Jones \& Boffin 2017). The mass-transfer between a close binary can lead to a bipolar outflow (Nordhaus \& Blackman 2006) and prolong strong magnetic fields by providing extra angular momentum (Tocknell et al. 2014). Since the first PN with a confirmed binary CS was discovered (Bond 1976), more than 60 binary CSs have been found, ${ }^{15}$ laying a solid foundation for a binary-interaction model.

Globular clusters (GCs) provide a unique environment to test this scenario. Stars of old populations (like those in GCs) with turnoff mass $\sim 0.8 M_{\odot}$ will have $\sim 0.5-0.53 M_{\odot}$ (Alves et al. 2000; Kalirai et al. 2009) remaining at the end of AGB phase. The evolutionary timescale is longer than $10^{5} \mathrm{yr}$ (Caloi 1989) for such a low-mass core; thus, any remnant ejected at the end of the AGB phase would have dispersed before the CS gets hot enough to ionize the expelled gas, making it impossible to form any PNe (Schoenberner 1983). Buzzoni et al. (2006) also argued that, below a critical mass of $\sim 0.52 M_{\odot}$, no PN events are expected to occur under the assumption of single-star evolution. Therefore, any detection of PNe in GCs suggests an

\footnotetext{
${ }^{15}$ http://www.drdjones.net/bCSPN/
} 
alternative evolutionary channel. The binary-interaction model may provide a plausible explanation because a commonenvelope interaction or even a stellar merger, which is favored by GCs' high-density environment, will accelerate the evolutionary process and likely form a PN with low-mass CSs.

Since the first PN belonging to a GC was found in 1928 in M15 (Pease 1928), just three more PNe in GCs have been detected in the Milky Way (MW) (Gillett et al. 1989; Jacoby et al. 1997). Meanwhile, there have been some serendipitous discoveries for $\mathrm{PNe}$ in extragalactic GCs (Minniti \& Rejkuba 2002; Bergond et al. 2006; Chomiuk et al. 2008; Larsen 2008). Peacock et al. (2012b) conducted a systematic survey of the giant elliptical galaxy NGC 4472 in the Virgo cluster using FLAMES/GIRAFFE at the Very Large Telescope and found zero $\mathrm{PNe}$ in $174 \mathrm{GCs}$. Another ground-based spectroscopic survey in M31 (Jacoby et al. 2013) discovered three candidate PNe in 274 GCs. However, Bond (2015) ruled out one object found by Jacoby et al. (2013) through Hubble Space Telescope (HST) snapshot imaging. The luminous [O III] emission makes it possible to detect PNe at a distance beyond the MW via a narrowband filter centered on the redshifted wavelength of the [O III] line (Jacoby et al. 1990; Theuns \& Warren 1997) or using a medium-resolution spectrograph. Low-mass X-ray binaries are another potential source of [O III] emission in GCs. X-ray and [O III] emission have previously been observed from a GC in NGC 4472 (Zepf et al. 2007) and in NGC 1399 (Irwin et al. 2010). These low-mass X-ray binaries can be distinguished from $\mathrm{PNe}$ through their broad [O III] emission line profiles.

The number of PNe in a stellar population per unit bolometric solar luminosity, $\alpha$, has been used to place a constraint on the proportion of stars that contribute to planetary nebula luminosity function (PNLF) cutoffs. Galaxies with integrated $B-V$ smaller than 0.8 mag are characterized by $\alpha \sim 3 \times 10^{-7} \mathrm{PN} / L_{\odot}$, with the spread in $\alpha$ increasing significantly as the color becomes redder with respect to the constant value observed in bluer objects (Peimbert 1990; Buzzoni et al. 2006). Measurements for $\alpha$ in GCs are limited. In Galactic GCs, $\alpha \sim 2.0 \times 10^{-7} \mathrm{PN} / L_{\odot}$ and, in NGC 4472, Peacock et al. (2012a) gave an upper limit of $\alpha \leqslant 8 \times 10^{-8} \mathrm{PN} / L_{\odot}$ based on a non-detection.

Given that the mean GC luminosity is $\sim 10^{5} L_{\odot}$, hundreds of GCs need to be surveyed to place interesting constraints on $\alpha$ in these environments. Here, we report a systematic survey for PNe within GCs in the Virgo cluster with a sample size that is more than five times larger than the previous largest survey (Jacoby et al. 2013). In Section 2, we describe our data and reduction procedures. In Section 3, we describe our PN detection and confirmation procedures. Then in Section 4, we discuss the choice of PNLF, and we use the detection limits for each GC to quantify their contribution to the estimation of $\alpha$. We also discuss implications for PN formation channels. Finally, we summarize our study in Section 5.

\section{Data Reductions}

This work is based on the spectroscopic data of GC candidates around the giant elliptical galaxies M87, M49, M86, and M84 in the Virgo cluster, which were collected as part of the Next Generation Virgo Cluster Survey (NGVS). It offers deep imaging data of the Virgo cluster within its virial radius $\left(\sim 104 \mathrm{deg}^{2}\right)$ using the MegaCam instrument on the Canada-France-Hawaii Telescope (Ferrarese et al. 2012). The selection of GCs around M87 and the reduction of their spectra were described in Zhang et al. (2015) and Ko et al. (2017). The primary selection for our GC targets is based on color. We also considered the concentration index as a secondary selection criterion. Since most of the GCs are unresolved at the distance of the Virgo cluster, it only biases the GC selection marginally based on their densities or sizes. The authors used the Hectospec multifiber spectrograph (Fabricant et al. 2005) on the $6.5 \mathrm{~m}$ MMT telescope to carry out an extensive spectroscopic survey of Virgo GCs and ultra-compact dwarfs (UCDs; $3650-9200 \AA, R \sim 1000$ ). The data were reduced through version 2.0 of HSRED reduction pipeline, ${ }^{16}$ which includes the basic corrections (bias, dark, and flat-fielding), aperture extraction, and wavelength calibration. We completed the flux calibration following the steps described in Fabricant et al. (2008), which include corrections for atmospheric extinction, Hectospec relative throughput, and absolute flux normalization with MegaCam $g^{\prime}$ photometry. The radial velocities were determined by a cross-correlation with SDSS stellar templates using the IRAF package RVSAO ${ }^{17}$ (Kurtz \& Mink 1998). As for the GCs around M49, they were collected and reduced following similar methods as used for the GCs in M87 (Y. Ko et al. 2019, in preparation). The median signal-to-noise ratio $(\mathrm{S} / \mathrm{N})$ per pixel is around 10 , with $\mathrm{S} / \mathrm{N}>5$ for more than $80 \%$ of our sample. The radial velocity uncertainties were derived from the correlation error, and their median velocity uncertainty is around $31 \mathrm{~km} \mathrm{~s}^{-1}$. In the following analysis, the spectra were shifted to the rest frame on the basis of the derived radial velocities correspondingly.

To avoid the contamination of foreground stars or background galaxies, we distinguished the GCs from contaminants based on their radial velocities and photometric information including concentration indices defined as the difference between 4 and 8 pix aperture-corrected magnitude, half-light radii, and colors. First, all of the spectroscopic targets with $v_{\mathrm{r}}>3000 \mathrm{~km} \mathrm{~s}^{-1}$ were considered background sources (see references in Ko et al. 2017). Second, we distinguished UCDs among our sample. We measure the half-light radii for the bright sources with $i<21.5 \mathrm{mag}$. The sources with half-light radii of $r_{\mathrm{h}}>11 \mathrm{pc}$ are considered as the UCDs. Third, for the remaining sources, we separate them into two groups through radial velocities. For the sources with radial velocities higher than $500 \mathrm{~km} \mathrm{~s}^{-1}$, we consider them as the GCs, because the Galactic stars rarely have those high radial velocities. For the sources with lower radial velocities, we adopt the extreme deconvolution algorithm (Bovy et al. 2011) on the multidimensional color space and the concentration index (see Section 2.3.1 in Longobardi et al. 2018). Based on this process, we remove foreground Galactic stars from our sample.

Instead of using five templates to detect the emission line features (Jacoby et al. 2013), we used simple stellar population (SSP) models of Bruzual \& Charlot (2003, hereafter BC03) adopting the Chabrier (2003) IMF with metallicity from $Z=0.0001$ to 0.05 and age from 1 to $10 \mathrm{Gyr}$. Ages and masses for these clusters were determined by comparison with models (e.g., Worthey 1994). The BC03 models do not include the emission lines of a possible PN; thus, a model-subtracted spectrum should preserve these emission lines. Some redundant continuum components remained after the subtraction of the

\footnotetext{
16 http://www.mmto.org/node/536

17 http://tdc-www.harvard.edu/iraf/rvsao
} 


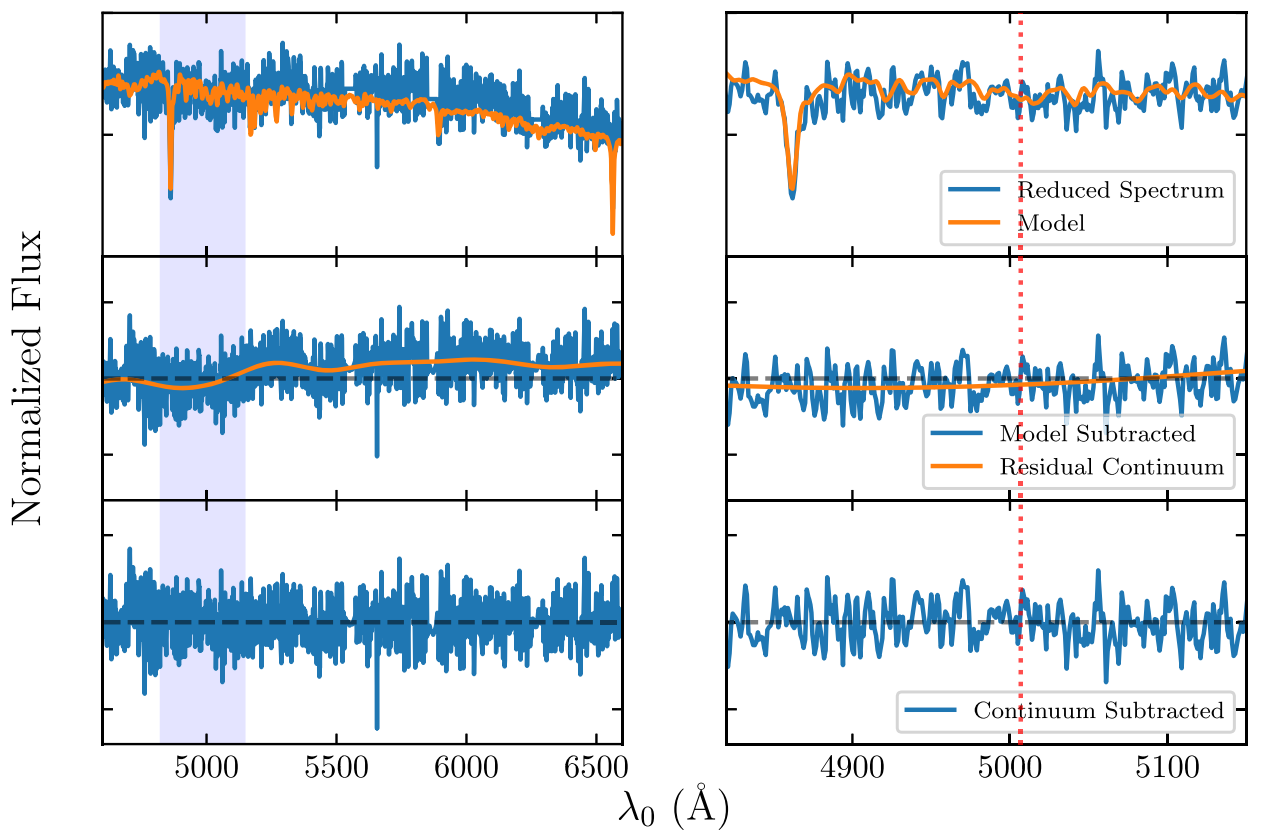

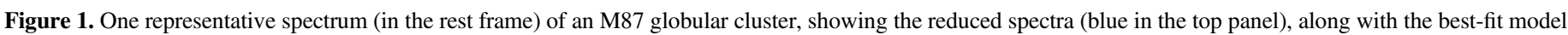

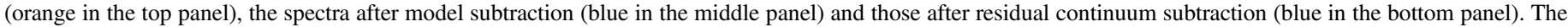

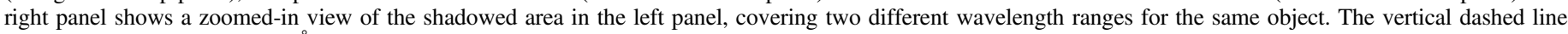
indicates the location of $5007 \AA$.

model spectrum (see the middle panels of Figure 1). Since they had little or no influence on the emission line features we were interested in, they were subtracted before we performed a $\chi^{2}$ test to derive the best-fit model. The calibrated spectra are present in the bottom panel of Figure 1 with a vertical dashed line indicating the location of $5007 \AA$. Through visual inspection, we verified that this procedure works well for most of our clusters.

\section{Finding Planetary Nebulae in GCs}

To verify the detection of PNe in GCs, we used three criteria. The emission line of [O III] $5007 \AA$ is the most notable feature of a PN, which makes it the most important criterion. In the second place, emission line profiles and flux ratios between the emission lines ([O III] $4959 \AA, 5007 \AA$ and $\mathrm{H} \alpha / \mathrm{H} \beta$ ) should be consistent with those expected from a PN. Last but not least, the radial velocity of a candidate, from its emission lines, must be consistent with its parent GC.

We searched for lines whose flux deviation from zero is larger than $3 \sigma$ where $\sigma$ is estimated from a part of the calibrated spectrum where no strong absorption or emission lines exist. If an emission line feature is spotted within 3 around $5007 \AA$, this might be a candidate PN. Note that the value of the typical error in redshift determination is around $31 \mathrm{~km} \mathrm{~s}^{-1}$ (corresponding to an error of $0.5 \AA$ in wavelength); therefore, it will not affect the detection for the [O III] 5007 emission from PNe. We also looked for the emission lines around $4959 \AA$, which is another noteworthy feature of PNe. The flux ratios among the emission lines have been proven to be an effective tool in identifying PNe. Arnaboldi et al. (2003) first used $I(\lambda 4959) / I(\lambda 5007)$ to confirm extragalactic PNe in the Virgo cluster. The flux ratio between 4959 and $5007 \AA$ should be close to one-third $(I(\lambda 4959) / I(\lambda 5007) \approx 1 / 3)$ and the $[\mathrm{O}$ III] $5007 \AA$ to $\mathrm{H} \beta$ ratio should larger than two $(I(\lambda 5007) / I(\mathrm{H} \beta) \geqslant 2)$ in the metal-poor environments of GCs (Jacoby et al. 2013). For most of our clusters, however, reliable detection of Balmer lines and the determination of flux ratio may suffer from low $\mathrm{S} / \mathrm{N}$. The width of $\lambda 5007$ should be identical to the spectral resolution, excluding the chance of being a supernova remnant or X-ray binary, which has much broader emission lines than any PN (Williams 1980).

To estimate the absolute magnitude of [O III] $\lambda 5007$, we first inferred the continuum brightness of each cluster from the MegaCam $g^{\prime}$ band magnitude. The monochromatic flux around $5007 \AA$ was then estimated by comparing the strength of the emission relative to the continuum, i.e., the equivalent width. This corrects for flux loss from the fiber aperture. After that, the flux was transformed into apparent [O III] $\lambda 5007$ magnitude according to the relationship adopted in Ciardullo et al. (1989):

$$
m_{5007}=-2.5 \log F_{5007}-13.74
$$

In this way, we built a connection between $m_{5007}$ and $m_{g^{\prime}}$ using the flux ratio between the emission line and the continuum, which obviates the problem of flux calibration for fiber spectrophotometry. GCs are classified into two groups, adopting the criteria used in Longobardi et al. (2018). Briefly, If the GCs are located within 10 effective radii, $R_{\mathrm{e}}$, of the galaxy and have the relative radial velocities to the galaxy within $3 \sigma_{g}$ where $\sigma_{g}$ is the galaxy velocity dispersion, they are considered members of that galaxy. The effective radius and velocity dispersion information of Virgo galaxies were adopted from the NGVS galaxy catalog (L. Ferrarese et al. 2019, in preparation) and the values compiled by the GOLDMine project, respectively. If there is no information about the radius and velocity dispersions for any galaxy, we used the fixed values of $R_{\mathrm{e}}=30^{\prime \prime}$ and $\sigma_{g}=50 \mathrm{~km} \mathrm{~s}^{-1}$. For the GCs associated with galaxies, the distances were assumed to be the same as their host galaxy, adopting the distance from 

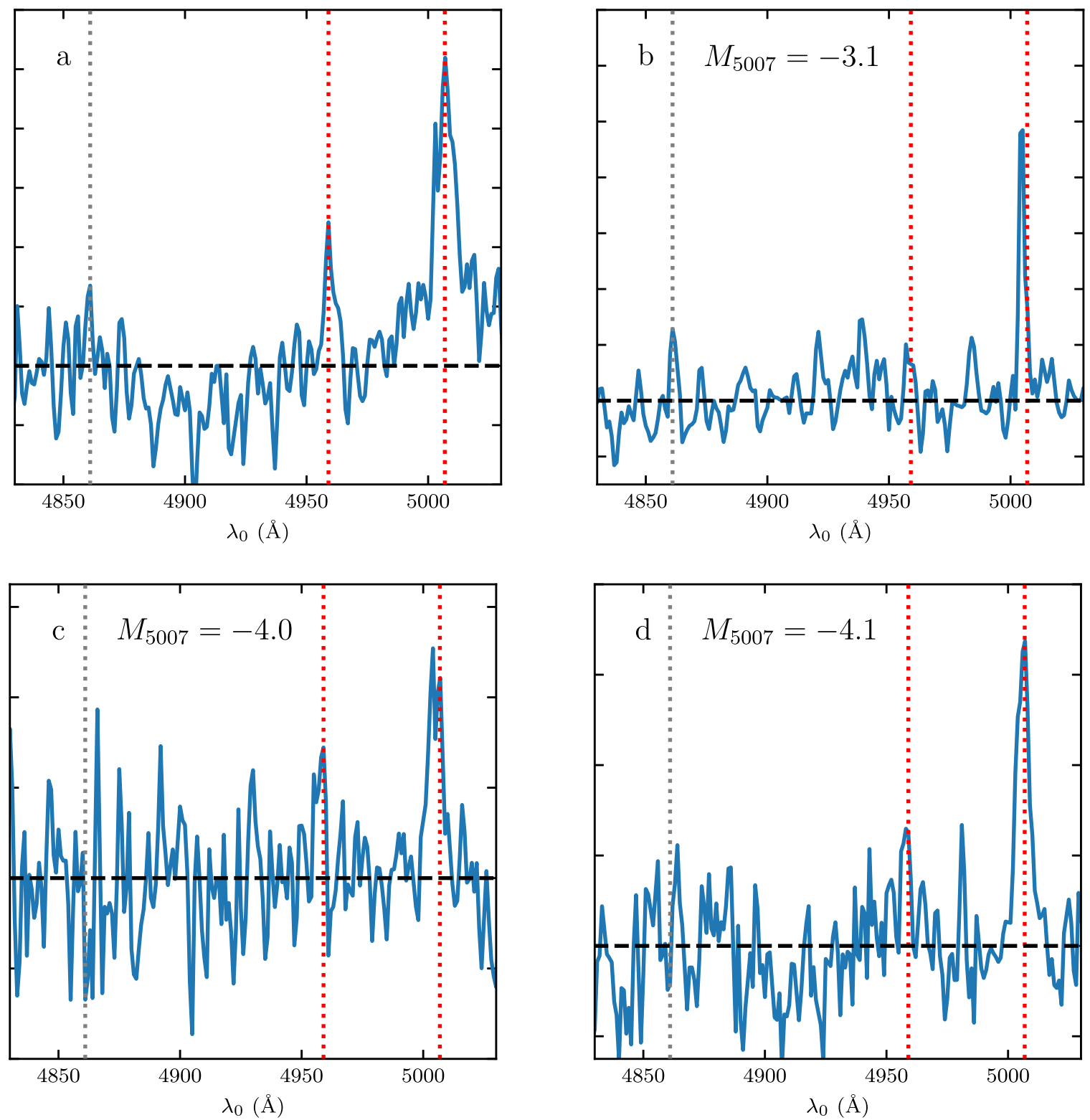

Figure 2. Spectra of four GCs with candidate PNe with emission lines around [O III] 4959, $5007 \AA$ with its identifier and the absolute magnitude of $5007 \AA$ emission (if available) in the middle of each figure. $M_{5007}$ of GC-c is calculated by summing up the flux in both peaks. The red vertical dashed lines represent [O III] 4959 , $5007 \AA$ and the gray vertical dashed line represents $\mathrm{H} \beta$ in the rest frame. The black horizontal dashed lines represent zero flux.

Blakeslee et al. (2009) and, for the intracluster GCs, the distances were taken as $16.5 \mathrm{Mpc}$ (Mei et al. 2007).

We report four GCs with nebular emission lines detected through this workflow. The spectra of these candidates are shown in Figure 2. We elaborate on their properties in the following paragraphs.

$G C-a$ (R.A. $=12^{\mathrm{h}} \cdot 28^{\mathrm{m}} \cdot 39^{\mathrm{s}} \cdot 69$, decl. $\left.=+7^{\circ} 53^{\prime} 32^{\prime \prime} \cdot 96\right)$. Also known as RZ 2109, this GCs was discovered to host the X-ray signature of an accreting stellar black hole (Maccarone et al. 2007). Follow-up studies confirmed the existence of strong and broad $\lambda 4959, \lambda 5007$ emission (Zepf et al. 2008; Peacock et al. 2012a) and attributed this emission to the photoionization of a strong wind driven from a stellar-mass black hole.

$G C-b$ (R.A. $=12^{\mathrm{h}} \cdot 29^{\mathrm{m}} \cdot 44^{\mathrm{s}} .51$, decl. $\left.=+8^{\circ} 0^{\prime} 299^{\prime \prime} 67\right)$. This cluster is classified as old $(\sim 10 \mathrm{Gyr})$ and metal-poor $(Z \sim 0.004)$ based on the comparison to the BC03 models. The radial velocity of the cluster is $970 \pm 11 \mathrm{~km} \mathrm{~s}^{-1}$, associated with M49. It has a solid detection of an emission feature around $5007 \AA$ with a significance of $\sim 9 \sigma$ while $\mathrm{H} \beta$ and $\lambda 4959$ are doubtful. Its absolute magnitude $M_{5007}$ is around $-3.1 \mathrm{mag}$, within the range of the PNLF. However, the center of the emission line is not aligned with $5007 \AA$ and has an offset of $2.3 \AA$ in the rest frame (corresponding to $\Delta v_{\mathrm{r}} \sim 140 \mathrm{~km} \mathrm{~s}^{-1}$ ), which casts doubt on its association with the GC. We adopted the same criterion as Jacoby et al. (2013) that the difference of radial velocity, $\Delta v_{\mathrm{r}}$, between emission lines (coming from the star) and absorption lines (coming from the cluster) should satisfy this relation $\Delta v_{\mathrm{r}} \leqslant 3 \sigma_{\text {eff }}$, where $\sigma_{\text {eff }}$ is the combination of the system's internal velocity dispersion and the uncertainty in radial velocity measurement. Its internal velocity dispersion was assumed to be a typical value of $10 \mathrm{~km} \mathrm{~s}^{-1}$, and the uncertainties of the radial velocity of the cluster and the emission line are $11 \mathrm{~km} \mathrm{~s}^{-1}$ and $7 \mathrm{~km} \mathrm{~s}^{-1}$, respectively. The overall $\sigma_{\text {eff }}$ is estimated to be $\sigma_{\text {eff }} \approx 17 \mathrm{~km} \mathrm{~s}^{-1}$. Therefore, we ruled out its 
association of this emission line with a GC. Given that it is close distance to the center of M49 (43". 5, Ferrarese et al. 2006), we attributed its source to be a superposition with a field $\mathrm{PNe}$ in the M49. To estimate the likelihood, we calculated the possibility of a field PNe being detected by one fiber in our survey. We adopted the best-fitting core-Sérsic surface brightness profile (Graham et al. 2003) for M49 from Ferrarese et al. (2006). The number of $\mathrm{PNe}$ is connected with the underlying stellar population through $\alpha$ :

$$
N_{\mathrm{PN}}=\alpha L_{\mathrm{bol}}
$$

where the $L_{\text {bol }}$ represents the bolometric luminosity. The bolometric correction is done via

$$
I=10^{-0.4\left(\mathrm{BC}_{V}-\mathrm{BC}_{\odot}\right)} 10^{-0.4(\mu-K)}
$$

with the solar bolometric correction $\mathrm{BC}_{\odot}=-0.07$, and $K=26.4$ mag $\operatorname{arcsec}^{-2} . \quad \mathrm{BC}_{V}$ is assumed to be fixed, $\mathrm{BC}_{V}=-0.85$, with $10 \%$ accuracy (Buzzoni et al. 2006). As for the $\alpha$, we adopted the value from Hartke et al. (2017), who conducted a photometric survey for PNe in the extended halo of M49. Even though their survey excluded objects within a major-axis radius of $159^{\prime \prime}$ and $\alpha$ might be lower close to the center, it is still a reasonable value to estimate the contribution from field PNe. We calculated the expected number of field PN in the aperture of each fiber (1."5 in diameter) by coincidence and derived the possibility of having detected one PN in our survey to be around $20 \%$, suggesting it is possible to detect a field $\mathrm{PN}$ by chance.

$G C-c$ (R.A. $=12^{\mathrm{h}} \cdot 24^{\mathrm{m}} \cdot 30^{\mathrm{s}} \cdot 69$, decl. $\left.=+12^{\circ} 54^{\prime} 3{ }^{\prime \prime} 94\right)$. The peculiar feature of this cluster is that it has double-peaked emission lines at both 5007 and $4959 \AA$. The red peaks lie at the exact wavelength of [O III] while the blue peaks are shifted by $\sim 2.4 \AA\left(140 \mathrm{~km} \mathrm{~s}^{-1}\right)$, which is larger than $3 \sigma_{\text {eff. }}$ Given its distance from the center of M84 $\left(\sim 8^{\prime}\right)$, it is unlikely to be a field PN superposed along the line of sight. A possible source is $\mathrm{H}$ II region associated with the NGC 4438-N4388-M86 complex, similar to what was found by Gerhard et al. (2002). Oosterloo \& van Gorkom (2005) found evidence of an $\mathrm{HI}$ cloud therein, suggesting neutral gas stripped from the cluster galaxies. Although the cluster is close to the extended ionized gas region (see Figure 9 in Boselli et al. 2018b), there is no sign of $\mathrm{H} \beta$ or $\mathrm{H} \alpha$ emission even after we stacked the spectra of surrounding clusters. In that case, it is hard to imagine that these two peaks, which have almost the same flux, come from completely different physical processes. It has been suggested that a double-peaked line profile may result from the expanding shell of a PN (e.g., Gesicki et al. 1998). Schönberner et al. (2010) argued that the peak line emission corresponds to the denser inner regions where the emission is high. In their models, the expansion rates derived from the line peak separations, have a weak dependence on the metallicity and may reach $50 \mathrm{~km} \mathrm{~s}^{-1}$. Despite the fact this value is smaller than what we found in this cluster $\left(70 \mathrm{~km} \mathrm{~s}^{-1}\right)$, it is possible that this emission comes from a fast expanding PN. Given that this process is still poorly understood and, for the sake of clarity, we excluded this candidate from future analysis.

$G C-d$. This cluster is located at R.A. $12^{\mathrm{h}} .32^{\mathrm{m}} .17^{\mathrm{s}} .72$, decl. $+12^{\circ} 06^{\prime} 25$ ". 42 (J2000) and has a radial velocity of $1328 \pm 34 \mathrm{~km} \mathrm{~s}^{-1}$. The best-fit model for this cluster has metallicity of $Z=0.0004$ and an age of $10 \mathrm{Gyr}$. [O III] $\lambda 5007$ is well-measured $(7 \sigma),[\mathrm{O}$ III] $\lambda 4959$ is marginally detectable
(2.4 $\sigma$ ), and no emission is found at $\mathrm{H} \beta$ and $\mathrm{H} \alpha$. The flux ratio between $\lambda 4959$ and $\lambda 5007$ is close to one-third. This cluster lies far away enough from any galaxy; thus, the likelihood of it being a superposed field PN is negligible. Since there is no ionized region close to this cluster, we attribute the emission to be from a PN. The radial velocity determined from the [O III] emission lines is close to that from the absorption lines within the uncertainties, suggesting an association with the GC.

To sum up, of the four candidates with emission around $5007 \AA$, we found one GC whose emission lines can be attributed to a PN. The flux ratio $I(\lambda 4959) / I(\lambda 5007)$ and radial velocity all support that the emission comes from a PN within this GC. Therefore, we report a single detection out of 1469 GCs in the Virgo cluster.

\section{Discussion}

\subsection{Cutoff Magnitude $\mathrm{M}_{*}$}

In this work, we adopted the standard luminosity function of PNe from Ciardullo et al. (1989) ${ }^{18}$ :

$$
N(M) \propto e^{0.307 M}\left(1-e^{3\left(M_{*}-M\right)}\right)
$$

where $M_{*}$ is the cutoff magnitude of the PNLF. For stellar populations that are more metal-rich than the Large Magellanic Cloud, they are confirmed to have the same value of the cutoff $M_{*} \sim-4.5 \mathrm{mag}$ (e.g., Ciardullo 2012). However, as the population becomes more metal-poor, it has been argued that the cutoff depends on the metallicity (Dopita et al. 1992; Schönberner et al. 2010). The detailed formation and evolution of a PN is regulated by the metallicity in some aspects. As the metallicity decreases, the cooling efficiency of the gas drops off, causing a higher electron temperature. It can also reduce the power of the stellar wind from the CS, which leads to a more dilute environment compared with their metal-rich counterparts. The radiation field and the luminosity of the CS are also influenced by the metal content. Based on the photoionization/radiation-hydrodynamics in PNe models, both Dopita et al. (1992) and Schönberner et al. (2010) found the [O III] emissivity has a strong dependence on the metallicity, i.e., the cutoff magnitude becomes fainter as the metallicity decreases, although there is some discrepancy toward the metal-rich end.

This dependence on metallicity is, to some extent, confirmed by the observations of extragalactic systems with known distances. By collecting the distance of 16 galaxies derived from Cepheid and Tip of the Red Giant Branch measurements, Ciardullo (2012) found that $M_{*}$ does fade in metal-poor populations (see their Figure 5), roughly following the prediction of Dopita et al. (1992) and Schönberner et al. (2010). But we remind the reader of the large measurement uncertainties in these metal-poor and low-mass systems due to the low number of $\mathrm{PNe}$ and poorly defined luminosity functions. The other caveat that may limit its validity for GCs is that the sample of Ciardullo (2012) only extends to SMC-like metal-poor systems, which is still more metal-rich than typical GCs $([\mathrm{Fe} / \mathrm{H}]=-1)$ by $0.5-1$ dex. If we are willing to extrapolate this relation (Equation (4.1) in Dopita et al. 1992)

\footnotetext{
18 But see also Mendez et al. (1993), Méndez et al. (2008), Longobardi et al (2013), and Rodríguez-González et al. (2015) for other representations of PNLF.
} 
to the lower metallicities, the cutoff is expected to be at least $1 \mathrm{mag}$ fainter. However, there is one PN born in a metal-poor environment that may not follow this prediction. As shown in Table 2, Ps 1 is a confirmed PN found in an extremely metalpoor GC. The expected cutoff for such a population is around $M_{*}=0.6 \mathrm{mag}$, which is marginally fainter than the observed magnitude of [O III] emission from this PN. This finding tentatively casts some doubt on the robustness of these models (at least in GC-like systems) and calls for a better theoretical explanation for the PNLF itself.

With regards to our sample in the Virgo cluster, $M_{5007}$ of the $\mathrm{PN}$ in GC-d (and GC-c if included) is brighter by 1.5-2 mag than the cutoff predicted by Dopita et al. (1992)'s model. Nevertheless, as discussed in Section 3, there is no explanation for the [O III] emission other than a $\mathrm{PN}$ in at least one cluster. Therefore, we do not rule out the existence of a PN in GC-d based on this fainter cutoff magnitude, but rather take it as the evidence to question the steepness of this correlation in extremely metal-poor environments. The binary formation channel is likely to dominate in a GC-like environment (see Section 4.6) and these coalesced binary stars, such as blue straggler stars, have been proposed to account for the brightest of PNe (Ciardullo et al. 2005). Unfortunately, none of the currently available models (including Dopita et al. 1992; Schönberner et al. 2010) include binaries to explain the cutoff of the PNLF. This may explain why the models fail to predict the cutoff in a GC-like population correctly, and our work, although just a single detection, might be helpful in constraining the evolution model of PN within the framework of binary interaction.

To provide a closer look at the influence of the cutoff, we consider two choices for the cutoff in the following analysis: $M_{*}=-4.5 \mathrm{mag}$ and $M_{*}=-4.1 \mathrm{mag}$ to represent the case whether the cutoff has a dependence on metallicity. The latter one is chosen to be the magnitude of the brightest PN in our sample.

\subsection{Detection Limit}

Before we draw any conclusion from this single detection, it is crucial to assess the significance of this finding. Compared with previous extragalactic surveys for PNe (Peacock et al. 2012b; Jacoby et al. 2013), this apparent deficiency (1 out of 1469) of PNe could be the intrinsic properties of Virgo GCs, or because most PNe are below our observational detection limits. A cluster whose spectrum is not good enough to detect even the brightest PNe should have zero weight in the estimation of the luminosity-specific frequency of PN. Therefore, we use the detection limit, i.e., the minimum emission flux required for detection for an individual GC's spectrum, to quantify the contribution of a single GC to the estimation of $\alpha$.

To estimate the detection limit, we added a few mock lines around $5007 \AA$ to check the intensity required to get detected. A Gaussian profile with $\sigma=1.8 \AA$, corresponding to the spectral resolution, was used to simulate a mock emission line. Figure 3 exhibits one mock line test with zoomed-in views at the top. The central wavelength of the mock line was shifted by several angstroms (shown as the blue boxes) to eliminate random effects (possible emission/absorption feature, uncertainty in radial velocity determination or detection failure). In the zoomed-in figures, the intensity of mock lines is increased by a factor of 1.5 , corresponding to red dotted curves. The mock lines are marked orange when they are detected. We then took the average value of the intensity needed for detection as the detection limit for each individual observation and transformed it into absolute magnitude $M_{\text {lim }}$ using the same method as for $M_{5007}$.

Details of the detection limits are present in Figure 4. Detection limits versus $g^{\prime}$ band magnitudes of GCs are shown in the bottom panel and we present the histogram of detection limits at the top. We found a rough correlation between the detection limit and the brightness of the cluster, where brighter clusters have brighter $M_{\text {lim }}$. Most GCs are brighter than the sky in the fiber, therefore we expect that the detection limits for more luminous GCs are brighter due to larger noise. It is noticeable, however, that there is a large scatter, which may result from variation in observing conditions. Only 4 and 27 GCs have detection limits brighter than the cutoff of $M_{*}=-4.5 \mathrm{mag}$ and $M_{*}=-4.1 \mathrm{mag}$, respectively, illustrating the capability of detecting PNe using our data.

\section{3. $\alpha$ for GCs in the Virgo Cluster}

We used Monte Carlo (MC) simulations to constrain the value of $\alpha$ based on the single detection. $\alpha$ represents the specific-luminosity frequency for $\mathrm{PNe}$ within the range of the PNLF. The faint end of cutoff of the PNLF is taken to be $8 \mathrm{mag}$ below $M_{*}$ following Henize \& Westerlund (1963)'s prediction. $\alpha$ was assumed to be identical for every cluster in our sample. Based on the best-fit BC03 model, we derived the bolometric luminosity for each GC and obtained an expected value for the number of PNe by multiplying the luminosity by $\alpha$. These simulated PNe were assigned with a certain brightness $M_{5007}$ following the PNLF. Then, we counted the number of clusters with simulated PNe which have $M_{5007}$ beyond their detection limits. Since we had one GC detected with a PN, we calculated the probability $p(\theta \mid \alpha)$ of a single-detection $\theta$ for a given $\alpha$ by repeating the above procedures 1000 times. Using Bayes' Theorem:

$$
p(\alpha \mid \theta)=\frac{p(\theta \mid \alpha) p(\alpha)}{p(\theta)}
$$

and assuming the prior to be a uniform distribution, we can calculate the probability distribution of $\alpha$ given a single detection $(p(\alpha \mid \theta))$.

We show the distribution of $p(\theta \mid \alpha)$ and $p(\alpha \mid \theta)$ based on MC simulations in Figure 5. In the left panel of the figure, we can see that as $\alpha$ increases, $p(\theta \mid \alpha)$ first increases from zero and reaches a maximum around 0.35 at $\alpha \sim 10^{-8} \mathrm{PN} / L_{\odot}$. The probability then decreases since more than one simulated $\mathrm{PNe}$ are detected in the GCs. In the right panel, the distribution of $p(\alpha \mid \theta)$ is presented in log-log space with the best-fit gamma distribution (dashed line) and the $68.3 \%$ confidence interval (shadowed area). The maximum-likelihood estimations of $p(\alpha \mid \theta)$ are around $3.0 \times 10^{-9} \mathrm{PN} / L_{\odot}$ and $3.9 \times 10^{-8} \mathrm{PN} / L_{\odot}$, with the $68.3 \%$ confidence interval from $2.0 \times 10^{-9} \mathrm{PN} / L_{\odot}$ to $6.6 \times$ $10^{-9} \mathrm{PN} / L_{\odot}$ from $3.2 \times 10^{-8} \mathrm{PN} / L_{\odot}$ to $9.1 \times 10^{-8} \mathrm{PN} / L_{\odot}$ for $M_{*}=-4.5 \mathrm{mag}$ and $M_{*}=-4.1 \mathrm{mag}$, respectively. If we include the cluster with double-peaked emission lines, GC-c, $\alpha$ is estimated to increase by a factor of 1.5 . See Table 1 for a summary of $\alpha$ in this work.

Peacock et al. (2012b) reported a non-detection result in M49 and they estimated the upper limit of $\alpha$ by combining the overall bolometric luminosity of all the GCs in their sample which gave a value of $\alpha \leqslant 8 \times 10^{-8} \mathrm{PN} / L_{\odot}$ (the upper limit is 

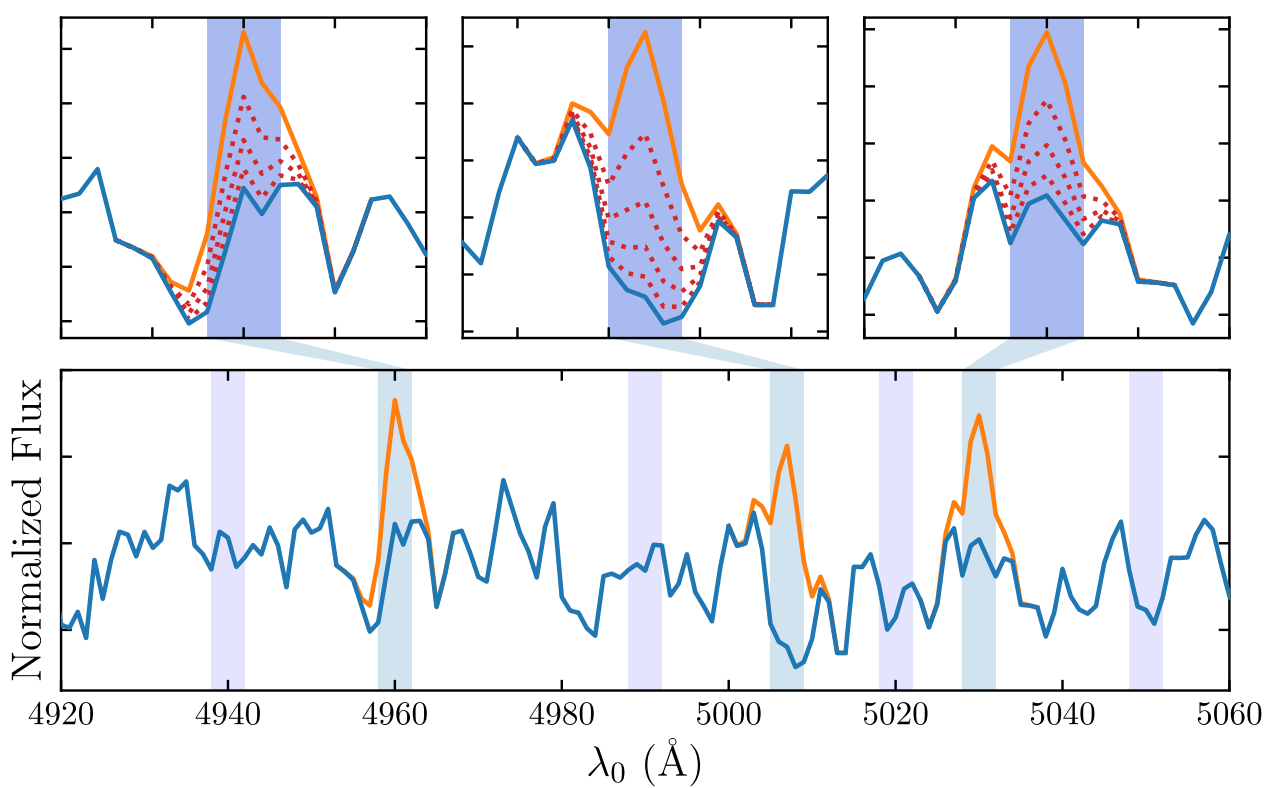

Figure 3. Mock line tests. A Gaussian-like profile is added to the calibrated spectrum at the specific wavelength. The intensity of the mock line increases until the new line can be detected by the code. The above process is applied at various wavelengths (shown as the blue boxes) to eliminate random effects. Zoomed-in spectra are shown at the top. Red dotted curves represent the spectra with mock lines added. The mock line profiles are marked orange when they are detected. The average value of the intensity needed for detection is taken as the detection limit for each individual observation.
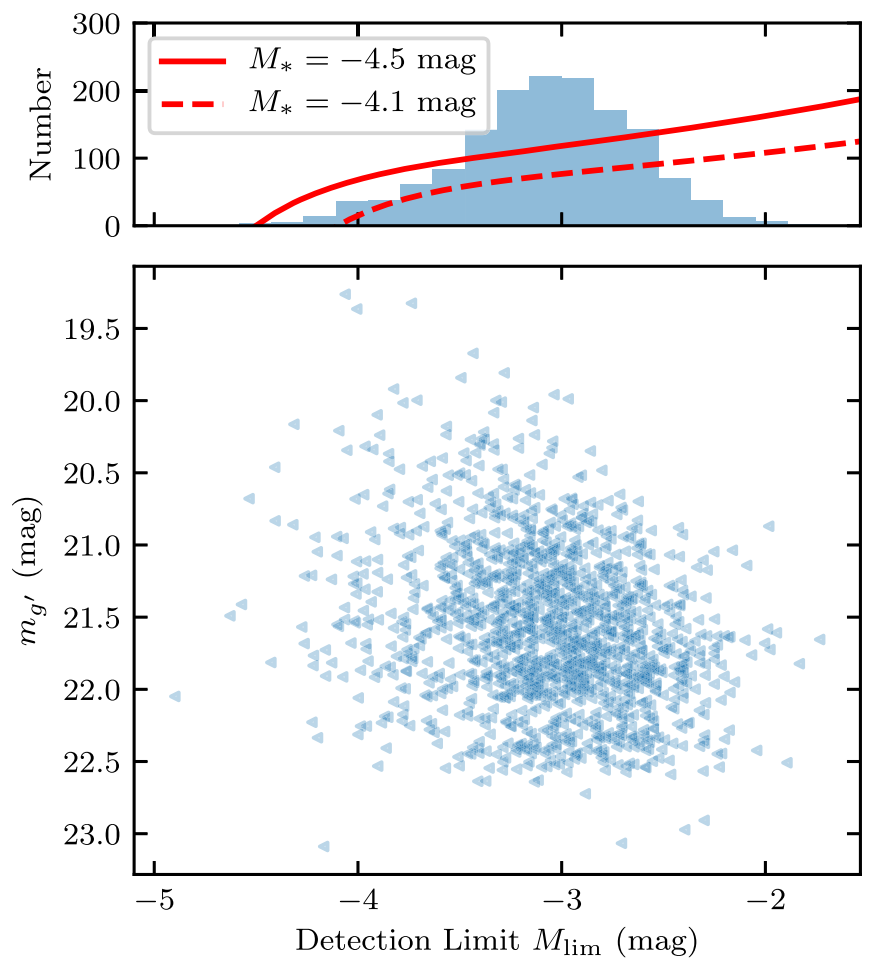

Figure 4. Detection limits vs. the $g^{\prime}$ band magnitudes of GCs. There is a rough correlation between the detection limit and the brightness of the cluster, in the sense that brighter clusters have brighter $M_{\text {lim }}$. In the top panel, the histogram of the detection limits are present, compared with the PNLF of different cutoff: $M_{*}=-4.5 \mathrm{mag}$ (red solid) and $M_{*}=-4.1 \mathrm{mag}$ (red dashed). Any cluster whose detection limit is brighter than the bright-end cutoff cannot have a single PN detected. Only 4 and 27 GCs have detection limits brighter than the cutoff of $M_{*}=-4.5 \mathrm{mag}$ and $M_{*}=-4.1 \mathrm{mag}$, respectively, illustrating the capability of detecting PNe using our data.

based on the $90 \%$ confidence level for a non-detection; Kraft et al. 1991). This upper limit is consistent with our result. Nevertheless, we point out their approach will underestimate the upper limit of $\alpha$ since the GCs with poor quality (bright detection limits) are also included in the total luminosity.

\section{4. $\alpha$ for UCDs in the Virgo Cluster}

Following the same procedures as for GCs, we also estimate $\alpha$ for UCDs in the Virgo cluster. UCDs are compact stellar systems that are brighter than most GCs but fainter than compact ellipticals. Francis et al. (2012) revealed that the UCDs are old and (generally) metal-rich (mean $[\mathrm{Fe} / \mathrm{H}]=$ $-0.8 \pm 0.1)$. The catalog of UCDs, which consists of 121 UCDs, was compiled from Liu et al. (2015), Zhang et al. (2015), and C. Liu et al. (2019, in preparation). After subtracting the best-fit SSP models from the spectra, we found no trace of [O III] emission lines in the UCDs. Then, based on the non-detection result, we calculated the probability distribution of $\alpha$ for UCDs using the same method described in Section 4.3 and estimated its upper limit to be $\alpha \leqslant 2.2 \times$ $10^{-7} \mathrm{PN} / L_{\odot}$ and $\alpha \leqslant 3.0 \times 10^{-7} \mathrm{PN} / L_{\odot}$ at $68.3 \%$ significance level for $M_{*}=-4.5 \mathrm{mag}$ and $M_{*}=-4.1 \mathrm{mag}$, respectively.

\subsection{Expected Value for $\alpha$}

The $\alpha$ for GCs in the Virgo cluster are much lower than any result in the literature, which raises the question of what is expected. Here, $\alpha$ denotes the luminosity-specific PN density within the whole range of the PNLF, which follows the relationship (Ciardullo et al. 2005):

$$
\alpha=B \cdot t \cdot f
$$

where $B$ is the population's luminosity-specific stellar evolutionary flux, $t$ is the mean timescale for the [O III]-bright stage, and $f$ is the percentage of main-sequence stars that evolve into PNe. $B$ is around $(1-2) \times 10^{-11} L_{\odot}^{-1} \mathrm{yr}^{-1}$ for old stellar populations and is independent of age and metallicity (Buzzoni et al. 2006). Based on the calculation of energy available to post-AGB stars, they also estimated that the upper limit of the 

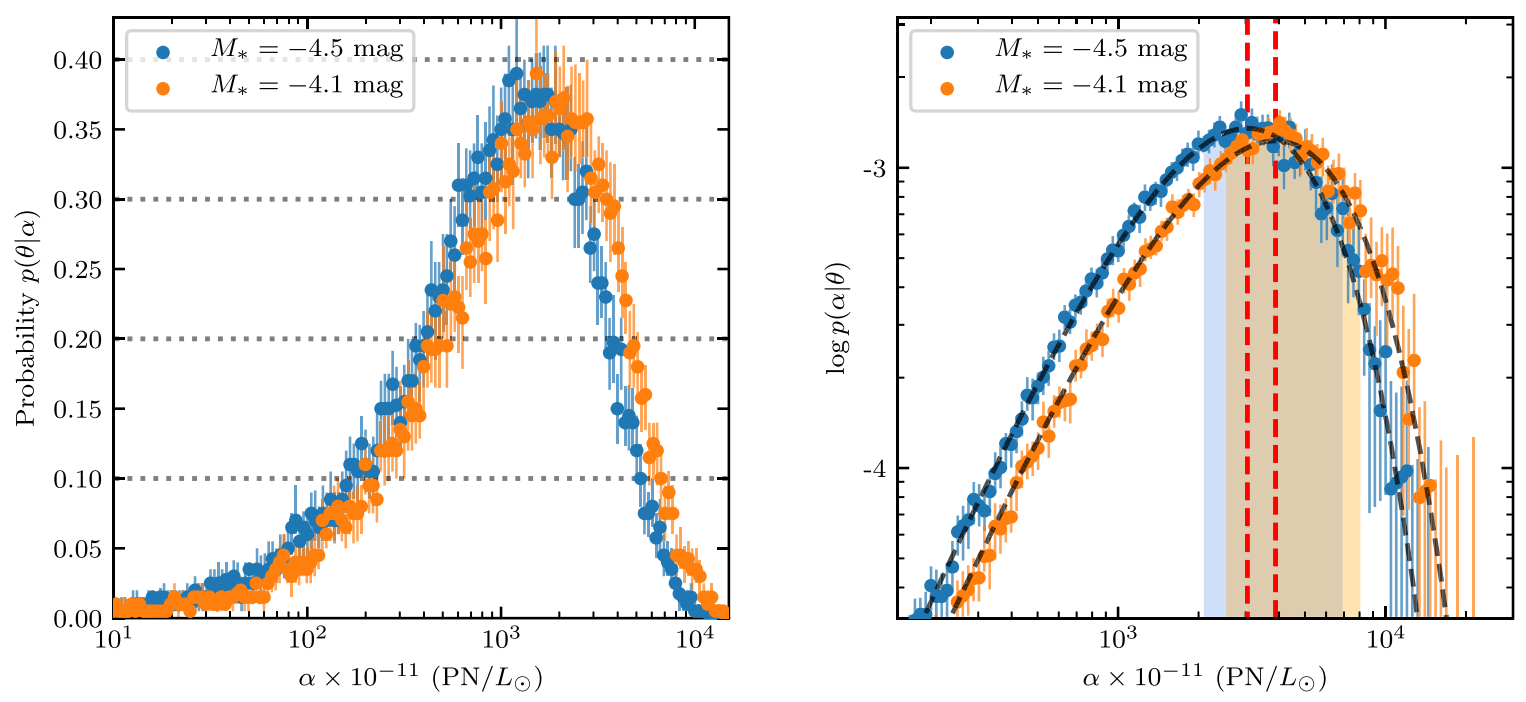

Figure 5. Probability of a single detection for various $\alpha$ on a log scale based on Monte Carlo (MC) simulations with different cutoff magnitude: $M_{*}=-4.5$ mag (blue) and $M_{*}=-4.1 \mathrm{mag}$ (orange). Each MC test was run 1000 times. Left panel: the probability distribution of a single detection at a given $\alpha(p(\theta \mid \alpha))$. Right panel: the probability distribution of $p(\alpha \mid \theta)$ calculated through Equation (5). The red vertical dashed lines represent the maximum-likelihood estimation $\left(3.0 \times 10^{-8} \mathrm{PN} / L_{\odot}\right.$ for $M_{*}=-4.5 \mathrm{mag}$ and $3.9 \times 10^{-8} \mathrm{PN} / L_{\odot}$ for $M_{*}=-4.1 \mathrm{mag}$ ) and the shadowed areas represent the $68.3 \%$ confidence interval (from $2.0 \times 10^{-8} \mathrm{PN} / L_{\odot}$ to $6.6 \times 10^{-8} \mathrm{PN} / L_{\odot}$ for $M_{*}=-4.5 \mathrm{mag}$ and from $3.2 \times 10^{-8} \mathrm{PN} / L_{\odot}$ to $9.1 \times 10^{-8} \mathrm{PN} / L_{\odot}$ for $\left.M_{*}=-4.1 \mathrm{mag}\right)$. The black dashed lines indicate the best-fit gamma distribution.

Table 1

$\alpha$ for Virgo GCs and UCDs

\begin{tabular}{lcc}
\hline \hline$\alpha\left(\mathrm{PN} / L_{\odot}\right)$ & $M_{*}=-4.5 \mathrm{mag}$ & $M_{*}=-4.1 \mathrm{mag}$ \\
\hline $\mathrm{GC}$ (single detection) & $3.0_{-1.0}^{+3.6} \times 10^{-8}$ & $3.9_{-0.7}^{+5.2} \times 10^{-8}$ \\
$\mathrm{GC}$ (two detections) & $4.6_{-1.4}^{+4.5} \times 10^{-8}$ & $5.9_{-1.8}^{+6.4} \times 10^{-8}$ \\
$\mathrm{UCD}$ (non-detection) & $\leqslant 2.2 \times 10^{-7}$ & $\leqslant 3.0 \times 10^{-7}$ \\
\hline
\end{tabular}

timescale of PNe should be shorter than $\sim 3 \times 10^{4} \mathrm{yr}$, which is its dynamical timescale. Thus, the measurement of $\alpha$ can provide a constraint on the percentage of stars that turn into PNe.

If all stars contribute to the $\operatorname{PNLF}(f=1)$ as the traditional scenario suggests, $\alpha$ should be smaller than $2 \times 10^{-11} \cdot 3 \times$ $10^{4} \sim 6 \times 10^{-7} \mathrm{PN} / L_{\odot}$. PNe, in reality, may have shorter lifetimes or lower transformation fractions; thus, $\alpha$ should be smaller than this maximum. Buzzoni et al. (2006) demonstrated that the timescale of $t$ should be extended with increasing age, due to the slow evolution of a low-mass star. However, it will reach a ceiling if the CSs take too long to get hot enough and ionize the nebular material. They concluded that any star with a CS mass smaller than $0.52 M_{\odot}$ will not be able to form a PN, which is an important limit for old populations like GCs.

The estimation mentioned above is based on the assumption of isolated stellar evolution. But it has been proposed that most $\mathrm{PNe}$ are generated through some kind of binary interaction (Moe \& De Marco 2006; Soker 2006). The binary hypothesis simply states that a majority of PNe have formed through a binary-interaction channel. Han et al. (1995) predicted that $38 \% \pm 4 \%$ of all PNe have been affected by binary interactions, and those from the post-common-envelope evolutionary channel account for one-third. Moe \& De Marco (2006) argued that $\sim 75 \%$ of the Galactic PNe can be ascribed to a common-envelope interaction. More than $40 \mathrm{PNe}$ have been associated with close binaries now (Miszalski et al. 2011). However, an overly tight binary system may also disrupt the formation of PNe. If the interaction occurs at early phases, such as during the red giant branch phase, the CS cannot ionize the outer layer that has been blown away. So not all binary systems can produce PNe. Moe \& De Marco (2006) argued that the separation limit for systems to form PNe should be between $100 R_{\odot}$ and $500 R_{\odot}$. Thus, in a GC-like system, $\alpha$ should be close to zero if we consider isolated stellar evolution. The binary-interaction scenario may indeed increase the value, but not significantly.

This low but nonzero value of $\alpha$ in Virgo GCs indicates that binary interaction might have an important role in forming $\mathrm{PNe}$ in GCs. Of the four Galactic GCs (GGCs) hosting PNe, two of them have a very high CS mass (Harrington \& Paltoglou 1993; Alves et al. 2000), which correspond to main-sequence masses of up to three times the cluster turnoff mass (Weidemann 2000). This could come from a mass-transfer binary or a merger formed in a dense GC environment that later becomes a PN. On the other hand, the low-mass CSs of an old population are intrinsically unable to generate any PNe. However, Jacoby et al. (2017) completed a full HST survey of all PNe in Galactic GCs, and they found only the CS of Ps 1 is higher than the predicted white-dwarf mass, thus requiring a history of mass augmentation (the $\mathrm{CS}$ of $\mathrm{JaFu} 1$ shows compact and bright [O III] and $\mathrm{H} \alpha$ emission, suggesting a binary companion).

In Table 2, we list all of the GC PNe that are currently published. The PNe span a wide range of [O III] luminosities, from close to the cutoff of the PNLF to 9 mag fainter. The PN we found in M87 is the brightest one ever found in a GC. Jacoby et al. (2013) noted that two GGCs with a PN also have a Low-mass X-ray binary (LMXB) embedded. Since the presence of an LMXB is connected to tight binary systems, this may suggest a possible binary origin for the GC PNe. However, by analyzing the stellar interaction rate in the dense cores of these clusters, Peacock et al. (2012b) concluded that no strong evidence exists. We matched our sample with the catalogs of known GCs that host LMXBs in the Virgo cluster (Maccarone et al. 2003; Jordán et al. 2004; Humphrey \& Buote 2008; Luan et al. 2018) and found 17 GCs containing an 
Table 2

Planetary Nebulae in Globular Clusters

\begin{tabular}{|c|c|c|c|c|c|}
\hline $\begin{array}{l}\text { Name } \\
\text { Host Galaxy/GC/PN }\end{array}$ & $\begin{array}{c}\operatorname{Age}^{a}(G C) \\
(G y r)\end{array}$ & $\begin{array}{l}M_{V}^{\mathrm{b}}(\mathrm{GC}) \\
\quad(\mathrm{mag})\end{array}$ & $\begin{array}{c}{[\mathrm{Fe} / \mathrm{H}]^{\mathrm{b}}(\mathrm{GC})} \\
(\mathrm{dex})\end{array}$ & $\begin{array}{c}M_{5007}(\mathrm{PN}) \\
(\mathrm{mag})\end{array}$ & References \\
\hline MW/M15/Ps 1 (K 648) & Old & -9.19 & -2.37 & 0.49 & Pease (1928) \\
\hline MW/M22/MRAS 18333 & Old & -8.50 & -1.70 & 5.04 & Gillett et al. (1989) \\
\hline $\mathrm{MW} / \mathrm{Pal}$ 6/JaFu 1 & Old & -6.79 & -0.91 & -2.8 & Jacoby et al. (1997) \\
\hline MW/NGC 6441/JaFu 2 & Old & -9.63 & -0.46 & 2.74 & Jacoby et al. (1997) \\
\hline NGC $3379 / g c 771 / P N$ & $\ldots$ & -8.65 & -1.40 & -1.62 & Bergond et al. (2006) \\
\hline Fornax-dSph/H5/PN & $\ldots$ & -7.40 & -1.73 & 1.33 & Larsen (2008) \\
\hline NGC 7457/GC7/PN & $\ldots$ & -8.20 & -0.40 & -3.74 & Chomiuk et al. (2008) \\
\hline M 31/B115-G117/PN & 14 & -8.54 & $0.1 \pm 0.1$ & -2.0 & Jacoby et al. (2013) \\
\hline M 31/BH16/PN & Old & -6.46 & -1.0 & 0.7 & Jacoby et al. (2013) \\
\hline $\mathrm{M} 31 / \mathrm{NB} 89 / \mathrm{PN}^{\mathrm{c}}$ & 10.4 & -6.60 & -0.6 & 0.5 & Jacoby et al. (2013) \\
\hline M 87/GC-d/PN & 10 & $-9.51^{\mathrm{d}}$ & -1.71 & -4.1 & this work \\
\hline
\end{tabular}

Notes.

${ }^{a}$ Ages were estimated by Caldwell et al. (2009, 2011).

${ }^{\mathrm{b}}$ For the Galactic GCs, these data were obtained from the Harris catalog 2010 edition (Shapley 1930; Harris \& Racine 1979; Harris 1996).

c Bond (2015) ruled out this one hosting a PN.

$\mathrm{d}$ The transformation relation is adopted from Betoule et al. (2013) and Jester et al. (2005).

LMXB. Given the field of view of Chandra X-ray observations, previous studies only targeted at the very center of the galaxies, which covers a fraction $(\leqslant 5 \%)$ of our sample. None of the GCs discussed in Section 3 were observed in these studies.

\section{6. $\alpha$ in GCs and Galaxies}

The $\alpha$ in GGCs is calculated to be $\alpha=2.0_{-0.6}^{+1.5} \times$ $10^{-7} \mathrm{PN} / L_{\odot}$ by summing the bolometric luminosity of GCs. The combined luminosity was estimated through the absolute $V$ band magnitude from Harris (1996) with bolometric corrections. Considering Jacoby et al. (1997)'s survey went 9 mag fainter than the cutoff, it is reasonable to assume the sample in Jacoby et al. (1997) to be complete even for the faintest PNe. Note that $\alpha$ for GGCs calculated in this method has no dependence on the choice of cutoff magnitudes. By comparing with that in GGCs, we found $\alpha$ in the Virgo cluster GCs is around 5-6 times smaller.

The binary fraction in the GCs could be the key to understand the difference. Since most GCs in our sample have $g^{\prime}$ band magnitude of $m_{g^{\prime}}<22.5 \mathrm{mag}$, which is $2-3 \mathrm{mag}$ brighter than the expected turnover of the GC luminosity function (GCLF; Harris 2001) at the distance of Virgo, they are more massive and denser compared with their Galactic counterparts. The "fundamental plane" of GCs shows that their size (half-light radius) has no dependence on their mass (McLaughlin 2000), which is also confirmed in Virgo cluster GCs (Jordán et al. 2005). An anticorrelation between cluster binary fraction with its absolute magnitude has been found in GGCs (Milone et al. 2012). Therefore, the Virgo GCs in our sample might be expected to have lower binary fractions, which may suggest a reduced efficiency in forming PNe if they are formed through a binary-interaction channel. To illustrate this effect, we divided GGCs into two groups: those brighter than the turnover luminosity and those fainter. Based on the detection of PNe in GGCs (three in bright GGCs and one in faint GGCs, see Table 2), we calculated $\alpha$ for the two groups, respectively. The results, together with Virgo GCs are shown in the left panel of Figure 6 with different colors denoting different groups. Because the two different cutoff magnitudes yield similar results for $\alpha$, in the following analysis, we use the more conservative choice of $M_{*}=-4.1 \mathrm{mag}$.

We see a trend that $\alpha$ decreases for brighter GCs, showing a similar tendency as the binary fraction (right panel). The effect of distance difference between Virgo GCs and GGCs has been taken into consideration in the Section 4.2 through the detection limit analysis. This suggests a connection between the efficiency of forming PNe in GCs with the binary fraction. Massive GCs with high stellar density may suppress the binary fraction due to the effect of binary disruption and therefore inhibit the formation of $\mathrm{PNe}$ from the binary-interaction channel. Another possible cause might be ram-pressure stripping by the intracluster medium. Villaver et al. (2003) shown that ram pressure can effectively alter the morphology of PNe and most of the mass ejected during the AGB phase is left downstream. However, we argue that this is probably not the underlying cause for two reasons. First, there are plenty of PNe outside of GCs in these massive galaxies, and the $\alpha$ measured in the field is higher than in GCs (see the following text and Figure 8). This is the reverse of what one might expect, as the field stars are more centrally concentrated than the GCs. Second, M49's $\alpha$ in the field is lower than M87's, which is the opposite of naive expectations for a ram-pressure mechanism. Another possible cause is an internal mechanism within GCs (e.g., collision or nearby stellar radiation) that may shorten the lifetime of PNe by disrupting the stellar ejecta.

If the binary fraction is the driving force behind the difference of $\alpha$ in different groups, as suggested by Ciardullo et al. (2005) and Jacoby et al. (2013), the formation efficiency of PNe is expected to correlate with the cluster mass or absolute luminosity, $M_{V}$. This correlation can be represented by a power-law relation between $\alpha$ and $M_{V}$ with an index of $0.25 \pm 0.08$. This relationship, however, is only valid if Virgo GCs and GGCs are intrinsically similar, with $\alpha$ largely driven by internal processes. It could instead be the case that the low $\alpha$ in Virgo GCs is due to their unique environment (i.e., in giant ellipticals or in a galaxy cluster), in which case we might expect $\alpha$ in fainter Virgo GCs to be similarly low. Below, we explore how a survey for PNe in fainter GCs can distinguish between these two cases. 

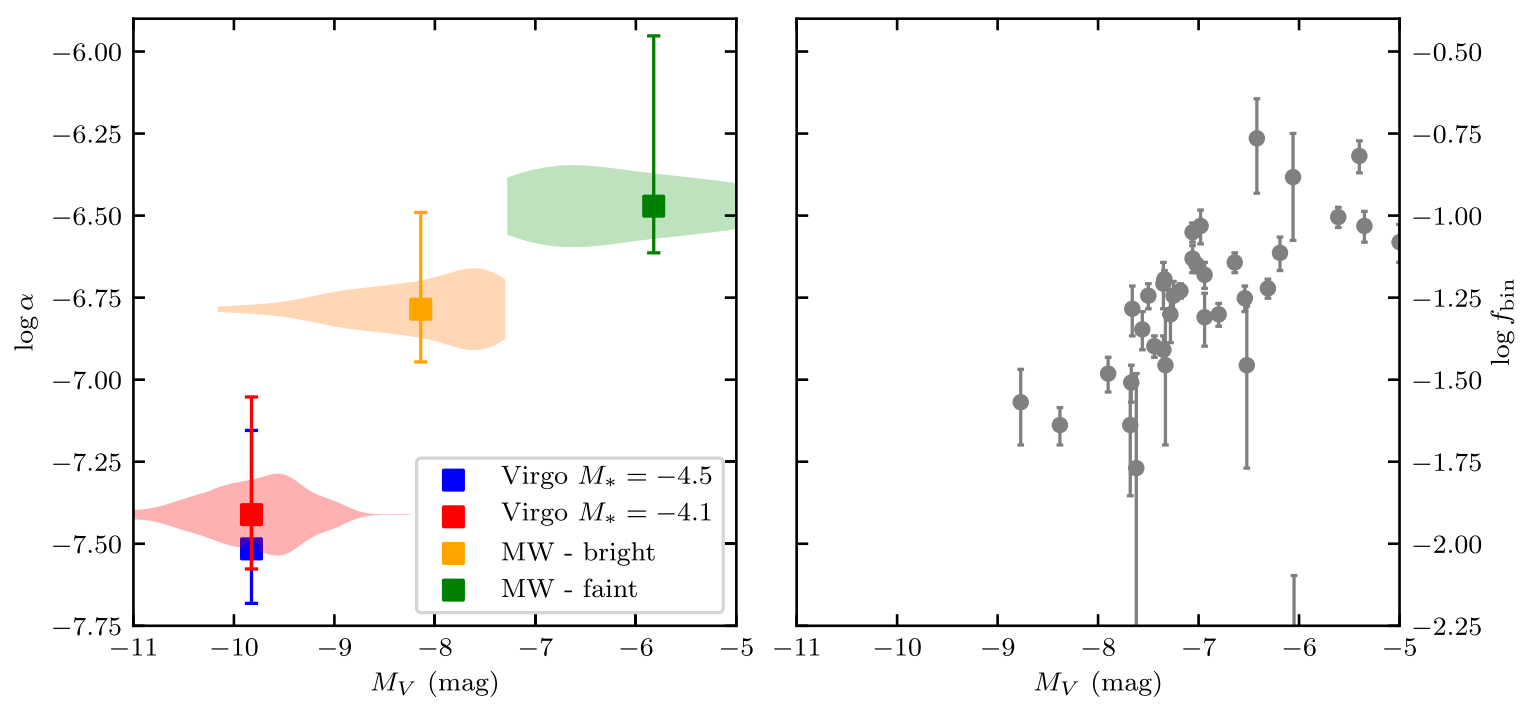

Figure 6. Left panel: number of PNe per bolometric luminosity $\alpha$ vs. absolute $V$ band magnitude of Virgo GCs $\left(M_{*}=-4.5\right.$ mag, blue, and $M_{*}=-4.1$ mag, red)), GGCs brighter than the turnover luminosity ( $M_{V}=-7.3 \mathrm{mag}$, orange), and GCCs fainter than the turnover luminosity (green). The values of $\alpha$ in GGCs do not rely on the choice of different cutoffs. The thickness of the horizontal violin plots represent the distribution of the brightness of GCs in each group. The error bars represent the 68.3\% confidence intervals of $\alpha$ for each group. Because the two different cutoff magnitudes yield similar results for $\alpha$, in the following analysis, we use the more conservative choice of $M_{*}=-4.1 \mathrm{mag}$. Right panel: fraction of binaries with $q \geqslant 0.5$ in the core (Milone et al. 2012) as a function of the absolute visual magnitude of the host GC in the Milky Way. Note that the scales of $\alpha$ and $f_{\text {bin }}$ are exactly the same. $\alpha$ decreases as the GCs becoming brighter, suggesting that $\alpha$ is influenced by an environmental factor in cluster, and the binary fraction could be the key to understand this correlation.

Case 1 adopts a correlation between $\alpha$ and $M_{V}$ as mentioned before, while case 2 considers a fixed $\alpha$ for all GCs, having no dependence on $M_{V}$. In a flux-limited survey, the effect of the GCLF should also be taken into consideration. Here, we adopt a symmetric Gaussian GCLF of $\sigma_{\mathrm{GCLF}}=1.37 \mathrm{mag}$ (Peng et al. 2009 ) and took the total number of GCs around the giant elliptical galaxies M87, M49, M86 and M84 $(29434 \pm 1715)$ from Peng et al. (2008). Moreover, since the sensitivity to PNe is mainly determined by the flux level of background (see Figure 4), sky background becomes the dominant component for noise and the detection limit should remain unchanged as the survey goes deeper. In Figure 7, we present the expected number of GCs hosting PNe detected per magnitude bin $\left(\Delta M_{V}=0.5 \mathrm{mag}\right)$. The blue and orange histograms represent the expected number of candidates per magnitude bin $\left(\Delta M_{V}=0.5 \mathrm{mag}\right)$ for case 1 and case 2 , respectively. The red vertical dashed line indicates the brightness of the faintest GCs targeted for spectroscopy in our survey.

We find a significant difference between the expected number for case 1 and case 2. For case 1, GCs hosting PNe are most likely to be discovered in clusters with absolute magnitude of $M_{V} \sim-7.7 \mathrm{mag}\left(m_{g^{\prime}} \sim 23.6 \mathrm{mag}\right)$ while the value for case 2 is 1.4 mag brighter. We predict that for every 0.5 mag down the GCLF that is surveyed, there will be more than two times more candidates detected for case 1 compared with case 2 . For case 1 , if a survey goes deeper by $1.5 \mathrm{mag}$ down the GCLF, $\sim 20 \mathrm{PNe}$ brighter than $M_{5007}<-2 \mathrm{mag}$. For case 2, however, we expected the number to be around 5. This suggests that this method can differentiate between these two cases effectively.

$\mathrm{PNe}$ populations are known to be correlated with their galaxy's properties. More massive, metal-rich galaxies tend to have lower $\alpha$ (Peimbert 1990; Hui et al. 1993; Ciardullo et al. 2005). Another anticorrelation is observed with the farultraviolet excess of a galaxy, where many extreme horizontal

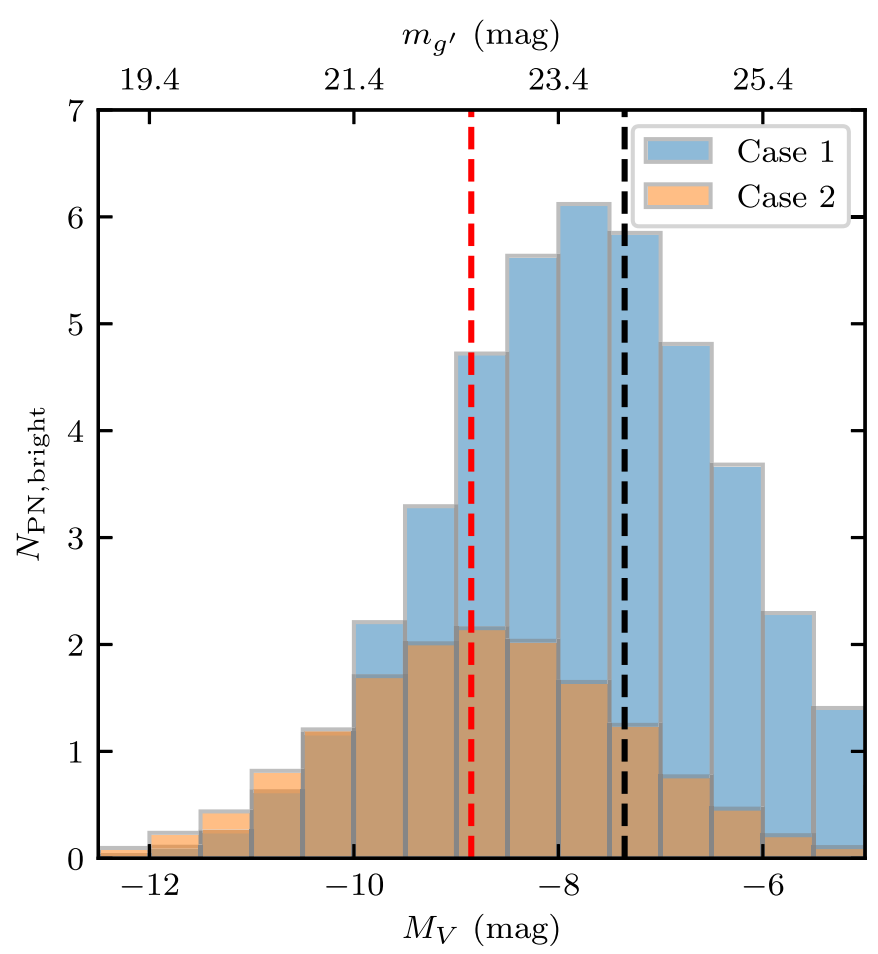

Figure 7. Expected number of GCs hosting bright $\mathrm{PNe}\left(M_{5007}<-2 \mathrm{mag}\right)$ detected per magnitude bin $\left(\Delta M_{V}=0.5 \mathrm{mag}\right)$. The blue and orange histograms represent the distribution of the number for case 1 and case 2, respectively. Case 1 adopts a correlation between $\alpha$ and $M_{V}$ in which $\alpha$ is largely driven by internal processes (binary fraction), while case 2 adopts the same $\alpha$ for all GCs, having no dependence on $M_{V}$. The red vertical dashed line indicates the faintest GCs targeted for spectroscopy in our survey. There is a significant difference between the expected number of PNe for case 1 and case 2 in a deeper survey. For case 1, if a survey goes deeper by $1.5 \mathrm{mag}$ down the GCLF (black vertical dashed line), $\sim 20$ bright PNe will be discovered. For case 2, however, we expect the number to be around 5. A deeper survey could be an effective method to differentiate between these two cases. 


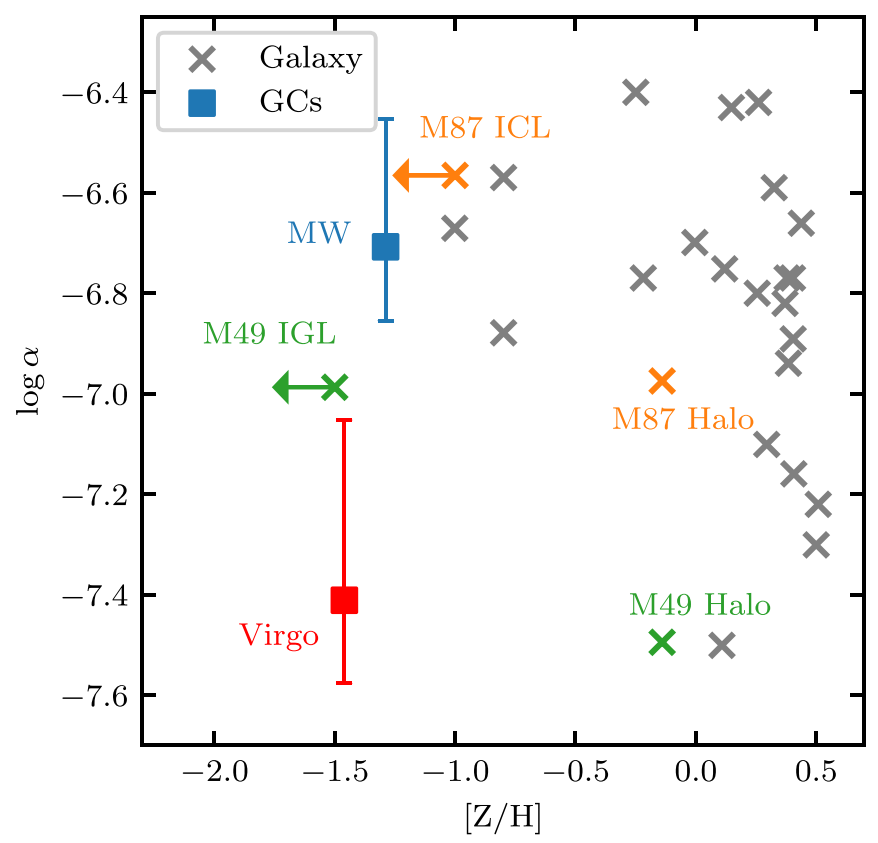

Figure 8. Number of PNe per unit bolometric luminosity $\alpha$ as a function of the host stellar population's metallicity. Crosses represent early-type galaxies, the MW bulge, LMC, and SMC. Filled squares show the GC systems. For Virgo $\mathrm{GCs}$, the cutoff is adopted as $-4.1 \mathrm{mag}$. Symbols with the same color are from the same galaxy but different environments (GCs, Halo, IGL, ICL).

branch stars will skip the AGB phase of stellar evolution entirely, reducing the number of PNe generated. Buzzoni et al. (2006) also pointed out a trend of $\alpha$ with galaxy color-a poorer PN population in redder ellipticals.

Figure 8 compares $\alpha$ for the MW's GC system, Virgo GC system, and a sample of galaxies (taken from Tables 4 and 6 of Buzzoni et al. 2006 which references $\alpha$ from Alloin et al. 1976; Jacoby 1980; Ciardullo et al. 1989; Hui et al. 1993; Jacoby \& De Marco 2002; Merrett et al. 2003; Buzzoni et al. 2006). The Virgo cluster has already been targeted by a number of PN surveys (Ciardullo et al. 1998; Feldmeier et al. 1998, 2004; Arnaboldi et al. 2002, 2003; Aguerri et al. 2005; CastroRodriguéz et al. 2009) to trace the faint diffuse stellar component that are not bound to individual galaxies. The $\alpha$ in the main galaxy halo and intracluster light (ICL) or intragroup light (IGL) of M87 and M49 were from Longobardi et al. (2015) and Hartke et al. (2018). The metallicities of M87's halo and ICL were adopted from Longobardi et al. (2015), and those of M49 were from Hartke et al. (2017; upper limit of metallicities in ICL/IGL). The metallicities of GCs in this work were calculated through the GC color-metallicity empirical relation adapted to Virgo galaxies M49 and M87 derived by Peng et al. (2006). The average values of metallicity were plotted in each GC system. $\alpha$ in four Galactic GCs is consistent with that in early-type galaxies, accompanied by a large uncertainty (due to the low number of known PNe), while in Virgo GC systems $\alpha$ has much lower estimates.

Thanks to the large number of GCs in our sample, we are able to derive a much tighter constraint on $\alpha$ than in previous studies. The correlation between $\alpha$ and the metallicity of the galaxies is clear; however, a turnover may exist toward lower metallicity. Unfortunately, the physics behind this correlation is not very clear. We believe the primary cause is increasing mass loss at high metallicities. Weiss \& Ferguson (2009) computed stellar evolution models of AGB stars with different metallicities and found the lifetime varies with metallicities, with the longest lifetime occurred for sub-solar metallicity. Rosenfield et al. (2014) also discovered a similar result, that lifetimes of low-mass AGB stars are expected to become shorter at decreasing metallicity $(Z \leqslant 0.008)$. These results may suggest that stellar populations with $[\mathrm{Fe} / \mathrm{H}]$ between -0.5 and -0.2 may have a larger value of $\alpha$ than their counterparts with higher or lower metallicity with the same luminosity.

\section{Conclusions}

We report the largest systematic survey for PNe in GCs. Our survey in the Virgo cluster, mainly around the giant elliptical galaxies M87, M49, and M86, consists of 1469 GCs in total. By subtracting the best-fit model from the spectra, we search for emission lines around $5007 \AA$ in the residual spectra. GC-d has an emission line at the exact wavelength of $5007 \AA$ with absolute magnitude $M_{5007}=-4.1 \mathrm{mag}$. The flux ratio $I$ $(\lambda 4959) / I(\lambda 5007)$ is close to one-third. We confirm that this is a GC hosting a PN and suggest it could be formed through binary interactions.

The [O III] emission is brighter than the cutoff predicted by Dopita et al. (1992) for extremely metal-poor population, suggesting that the dependence on the metallicity could be weaker than was expected. To estimate the specific-luminosity frequency of PNe $\alpha$, we use the detection limit to quantify the contribution of each GC in the calculation of $\alpha$ and $\mathrm{MC}$ simulations to constrain the probability distribution of $\alpha$ based on a single detection. The probability distribution $p(\alpha \mid \theta)$ is well fit by a gamma distribution and $\alpha \sim$ $3.0_{-1.0}^{+3.6} \times 10^{-8} \mathrm{PN} / L_{\odot}$ and $\alpha \sim 3.9_{-0.7}^{+5.2} \times 10^{-8} \mathrm{PN} / L_{\odot}$ for $M_{*}=-4.5 \mathrm{mag}$ and $M_{*}=-4.1 \mathrm{mag}$, respectively, which is at least 5-6 times lower than $\alpha$ in the Galactic GC system. $\alpha$ decreases toward brighter and more massive clusters, sharing a similar trend as the binary fraction in MW GCs (e.g., Milone et al. 2012). The discrepancy between the Virgo and Galactic GCs can be explained by the observational bias in extragalactic surveys toward brighter GCs. This low but nonzero efficiency is consistent with a binary origin for these PNe in GCs. The importance of CS binarity in the GC environment here is that binary interactions or mergers could form a blue straggler star that later becomes a PN with a more massive CS compared to the turnoff stars in the cluster. We predict the expected number of PNe within GCs to be detected in a deeper survey under two cases (mainly driven by internal processes or by external environment) and show that a deeper survey could be an effective method to differentiate between these two cases. Combined with $\alpha$ reported in other environments (GCs and elliptical galaxies), there may be a turnover of $\alpha$ at sub-solar metallicities.

We are grateful to the referees for their constructive input. E.W.P. acknowledges support from the National Natural Science Foundation of China under grant No. 11573002. A. L. acknowledges the support by the Centre national d'études spatiales. H.X.Z. acknowledges support from the CAS Pioneer Hundred Talents Program and the NSFC grant 11421303. M.G.L. and Y.K. acknowledge support from the National Research Foundation of Korea (NRF) grant funded by the Korean Government (NRF-2017R1A2B4004632) and the K-GMT Science Program (PID: 14A-MMT003/2014A-UAOG18) funded through the Korean GMT Project operated by the Korea Astronomy and Space Science Institute (KASI). I.C. is 
supported by Smithsonian Astrophysical Observatory Telescope Data Center. I.C. acknowledges additional support from the Russian Science Foundation grant 17-72-20119 and from the Program of development at M.V. Lomonosov Moscow State University through Leading Scientific School "Physics of stars, relativistic objects and galaxies." We thank the staff of the MMT Observatory for their professional support of our program. This research uses data obtained through the Telescope Access Program (TAP), which has been funded by the National Astronomical Observatories, Chinese Academy of Sciences, and the Special Fund for Astronomy from the Ministry of Finance. Based on observations obtained with MegaPrime/MegaCam, a joint project of CFHT and CEA/ DAPNIA, at the Canada-France-Hawaii Telescope (CFHT) which is operated by the National Research Council (NRC) of Canada, the Institut National des Sciences de l'Univers of the Centre National de la Recherche Scientifique of France, and the University of Hawaii. Observations reported here were obtained at the MMT Observatory, a joint facility of the University of Arizona and the Smithsonian Institution.

Software: Astropy (Astropy Collaboration et al. 2013), Matplotlib (Hunter 2007), RVSAO (Kurtz \& Mink 1998).

\section{ORCID iDs}

Weijia Sun (16) https://orcid.org/0000-0002-3279-0233 Eric W. Peng (i) https://orcid.org/0000-0002-2073-2781 Youkyung Ko 이 https://orcid.org/0000-0001-6333-599X Patrick Côté (10) https://orcid.org/0000-0003-1184-8114 Laura Ferrarese (1) https://orcid.org/0000-0002-8224-1128 Myung Gyoon Lee (1) https://orcid.org/0000-0003-2713-6744 Chengze Liu (i) https://orcid.org/0000-0002-4718-3428 Alessia Longobardi (i) https://orcid.org/0000-0001-5569-6584 Igor V. Chilingarian (1) https://orcid.org/0000-00027924-3253

Chelsea Spengler (10 https://orcid.org/0000-0002-1685-4284 Ann I. Zabludoff (1) https://orcid.org/0000-0001-6047-8469 Hong-Xin Zhang (1) https://orcid.org/0000-0003-1632-2541

\section{References}

Abell, G. O., \& Goldreich, P. 1966, PASP, 78, 232

Aguerri, J. A. L., Gerhard, O. E., Arnaboldi, M., et al. 2005, AJ, 129, 2585 Alloin, D., Cruz-González, C., \& Peimbert, M. 1976, ApJ, 205, 74 Alves, D. R., Bond, H. E., \& Livio, M. 2000, AJ, 120, 2044

Arnaboldi, M., Aguerri, J. A. L., Napolitano, N. R., et al. 2002, AJ, 123, 760 Arnaboldi, M., Freeman, K. C., Okamura, S., et al. 2003, AJ, 125, 514

Astropy Collaboration, Robitaille, T. P., Tollerud, E. J., et al. 2013, A\&A, 558, A33

Bergond, G., Zepf, S. E., Romanowsky, A. J., et al. 2006, A\&A, 448, 155

Betoule, M., Marriner, J., Regnault, N., et al. 2013, A\&A, 552, A124

Blakeslee, J. P., Jordán, A., Mei, S., et al. 2009, ApJ, 694, 556

Bond, H. E. 1976, PASP, 88, 192

Bond, H. E. 2015, AJ, 149, 132

Boselli, A., Fossati, M., Ferrarese, L., et al. 2018b, A\&A, 614, A56 Bovy, J., Hennawi, J. F., Hogg, D. W., et al. 2011, ApJ, 729, 141 Bruzual, G., \& Charlot, S. 2003, MNRAS, 344, 1000

Buzzoni, A., Arnaboldi, M., \& Corradi, R. L. M. 2006, MNRAS, 368, 877

Caldwell, N., Harding, P., Morrison, H., et al. 2009, AJ, 137, 94

Caldwell, N., Schiavon, R., Morrison, H., et al. 2011, AJ, 141, 61

Caloi, V. 1989, A\&A, 221, 27

Castro-Rodriguéz, N., Arnaboldi, M., Aguerri, J. A. L., et al. 2009, A\&A, 507, 621

Chabrier, G. 2003, PASP, 115, 763

Chomiuk, L., Strader, J., \& Brodie, J. P. 2008, AJ, 136, 234

Ciardullo, R. 2012, Ap\&SS, 341, 151
Ciardullo, R., Jacoby, G. H., Feldmeier, J. J., et al. 1998, ApJ, 492, 62 Ciardullo, R., Jacoby, G. H., Ford, H. C., et al. 1989, ApJ, 339, 53

Ciardullo, R., Sigurdsson, S., Feldmeier, J. J., et al. 2005, ApJ, 629, 499 De Marco, O. 2009, PASP, 121, 316

Dopita, M. A., Jacoby, G. H., \& Vassiliadis, E. 1992, ApJ, 389, 27 Fabricant, D., Fata, R., Roll, J., et al. 2005, PASP, 117, 1411

Fabricant, D. G., Kurtz, M. J., Geller, M. J., et al. 2008, PASP, 120, 1222

Feldmeier, J. J., Ciardullo, R., \& Jacoby, G. H. 1998, ApJ, 503, 109

Feldmeier, J. J., Ciardullo, R., Jacoby, G. H., et al. 2004, ApJ, 615, 196

Ferrarese, L., Côté, P., Cuillandre, J.-C., et al. 2012, ApJS, 200, 4

Ferrarese, L., Côté, P., Jordán, A., et al. 2006, ApJS, 164, 334

Francis, K. J., Drinkwater, M. J., Chilingarian, I. V., et al. 2012, MNRAS, 425,325

García-Segura, G., Langer, N., Różyczka, M., et al. 1999, ApJ, 517, 767

García-Segura, G., López, J. A., \& Franco, J. 2005, ApJ, 618, 919

García-Segura, G., Villaver, E., Langer, N., et al. 2014, ApJ, 783, 74

Gerhard, O., Arnaboldi, M., Freeman, K. C., et al. 2002, ApJL, 580, L121

Gesicki, K., Zijlstra, A. A., Acker, A., et al. 1998, A\&A, 329, 265

Gillett, F. C., Jacoby, G. H., Joyce, R. R., et al. 1989, ApJ, 338, 862

Graham, A. W., Erwin, P., Trujillo, I., et al. 2003, AJ, 125, 2951

Han, Z., Eggleton, P. P., Podsiadlowski, P., et al. 1995, MNRAS, 277, 1443

Harrington, J. P., \& Paltoglou, G. 1993, ApJL, 411, L103

Harris, W. E. 1996, AJ, 112, 1487

Harris, W. E. 2001, Saas-fee Advanced Course 28: Star Clusters (Berlin: Springer-Verlag), 223

Harris, W. E., \& Racine, R. 1979, ARA\&A, 17, 241

Hartke, J., Arnaboldi, M., Gerhard, O., et al. 2018, A\&A, 616, A123

Hartke, J., Arnaboldi, M., Longobardi, A., et al. 2017, A\&A, 603, A104

Henize, K. G., \& Westerlund, B. E. 1963, ApJ, 137, 747

Hui, X., Ford, H. C., Ciardullo, R., et al. 1993, ApJ, 414, 463

Humphrey, P. J., \& Buote, D. A. 2008, ApJ, 689, 983

Hunter, J. D. 2007, CSE, 9, 90

Irwin, J. A., Brink, T. G., Bregman, J. N., et al. 2010, ApJL, 712, L1 Jacoby, G. H. 1980, ApJS, 42, 1

Jacoby, G. H., Ciardullo, R., De Marco, O., et al. 2013, ApJ, 769, 10

Jacoby, G. H., Ciardullo, R., \& Ford, H. C. 1990, ApJ, 356, 332

Jacoby, G. H., \& De Marco, O. 2002, AJ, 123, 269

Jacoby, G. H., De Marco, O., Davies, J., et al. 2017, ApJ, 836, 93

Jacoby, G. H., Morse, J. A., Fullton, L. K., et al. 1997, AJ, 114, 2611 Jester, S., Schneider, D. P., Richards, G. T., et al. 2005, AJ, 130, 873 Jones, D., \& Boffin, H. M. J. 2017, NatAs, 1, 117

Jordán, A., Côté, P., Blakeslee, J. P., et al. 2005, ApJ, 634, 1002

Jordán, A., Côté, P., Ferrarese, L., et al. 2004, ApJ, 613, 279

Kalirai, J. S., Saul Davis, D., Richer, H. B., et al. 2009, ApJ, 705, 408

Ko, Y., Hwang, H. S., Lee, M. G., et al. 2017, ApJ, 835, 212

Kraft, R. P., Burrows, D. N., \& Nousek, J. A. 1991, ApJ, 374, 344

Kurtz, M. J., \& Mink, D. J. 1998, PASP, 110, 934

Larsen, S. S. 2008, A\&A, 477, L17

Liu, C., Peng, E. W., Côté, P., et al. 2015, ApJ, 812, 34

Longobardi, A., Arnaboldi, M., Gerhard, O., et al. 2013, A\&A, 558, A42

Longobardi, A., Arnaboldi, M., Gerhard, O., et al. 2015, A\&A, 579, A135

Longobardi, A., Peng, E. W., Côté, P., et al. 2018, ApJ, 864, 36

Luan, L., Jones, C., Forman, W. R., et al. 2018, ApJ, 862, 73

Maccarone, T. J., Kundu, A., \& Zepf, S. E. 2003, ApJ, 586, 814

Maccarone, T. J., Kundu, A., Zepf, S. E., et al. 2007, Natur, 445, 183

McLaughlin, D. E. 2000, ApJ, 539, 618

Mei, S., Blakeslee, J. P., Côté, P., et al. 2007, ApJ, 655, 144

Mendez, R. H., Kudritzki, R. P., Ciardullo, R., et al. 1993, A\&A, 275, 534

Méndez, R. H., Teodorescu, A. M., Schönberner, D., et al. 2008, ApJ, 681, 325

Merrett, H. R., Kuijken, K., Merrifield, M. R., et al. 2003, MNRAS, 346, L62

Milone, A. P., Piotto, G., Bedin, L. R., et al. 2012, A\&A, 540, A16

Minniti, D., \& Rejkuba, M. 2002, ApJL, 575, L59

Miszalski, B., Acker, A., Parker, Q. A., et al. 2011, in Asymmetric Planetary Nebulae 5 Conf., ed. A. A. Zijlstra et al. (Manchestser: Jodrell Bank Centre for Astrophysics), 109

Moe, M., \& De Marco, O. 2006, ApJ, 650, 916

Nordhaus, J., \& Blackman, E. G. 2006, MNRAS, 370, 2004

Nordhaus, J., Blackman, E. G., \& Frank, A. 2007, MNRAS, 376, 599

Oosterloo, T., \& van Gorkom, J. 2005, A\&A, 437, L19

Peacock, M. B., Zepf, S. E., Kundu, A., et al. 2012a, ApJ, 759, 126

Peacock, M. B., Zepf, S. E., \& Maccarone, T. J. 2012b, ApJ, 752, 90 Pease, F. G. 1928, PASP, 40, 342

Peimbert, M. 1990, RPPh, 53, 1559

Peng, E. W., Jordán, A., Blakeslee, J. P., et al. 2009, ApJ, 703, 42

Peng, E. W., Jordán, A., Côté, P., et al. 2006, ApJ, 639, 95 
Peng, E. W., Jordán, A., Côté, P., et al. 2008, ApJ, 681, 197

Rodríguez-González, A., Hernández-Martínez, L., Esquivel, A., et al. 2015, A\&A, 575, A1

Rosenfield, P., Marigo, P., Girardi, L., et al. 2014, ApJ, 790, 22

Sahai, R., \& Trauger, J. T. 1998, AJ, 116, 1357

Schoenberner, D. 1983, ApJ, 272, 708

Schönberner, D., Jacob, R., Sandin, C., et al. 2010, A\&A, 523, A86

Shapley, H. 1930, Star Clusters (New York: McGraw-Hill)

Soker, N. 1997, ApJS, 112, 487

Soker, N. 2006, ApJL, 645, L57
Theuns, T., \& Warren, S. J. 1997, MNRAS, 284, L11

Tocknell, J., De Marco, O., \& Wardle, M. 2014, MNRAS, 439, 2014

Villaver, E., García-Segura, G., \& Manchado, A. 2003, ApJL, 585, L49

Weidemann, V. 2000, A\&A, 363, 647

Weiss, A., \& Ferguson, J. W. 2009, A\&A, 508, 1343

Williams, R. E. 1980, ApJ, 235, 939

Worthey, G. 1994, ApJS, 95, 107

Zepf, S. E., Maccarone, T. J., Bergond, G., et al. 2007, ApJL, 669, L69

Zepf, S. E., Stern, D., Maccarone, T. J., et al. 2008, ApJL, 683, L139

Zhang, H.-X., Peng, E. W., Côté, P., et al. 2015, ApJ, 802, 30 